\title{
An Unusual Case of Extra-Enteric Blastocystosis in the Uterine Cervix
}

\author{
Yolanda Escutia-Guzman', Williams Arony Martinez-Flores², Joel Martinez-Ocaña², Ramon Martinez-Pimentel ${ }^{3}$, \\ Marisol Benitez-Ramirez', Fernando Martinez-Hernandez², Sara Arroyo-Escalante², Mirza Romero-Valdovinos², \\ Guadalupe Erendira Orozco-Mosqueda ${ }^{4}$, Pablo Maravilla ${ }^{2, *}$ [i] \\ 'Coordinacion de Cancer Cervicouterino, Laboratorio Estatal de Salud Publica, Morelia 58270, Michoacan, Mexico; ${ }^{2}$ Hospital General "Dr. Manuel \\ Gea Gonzalez", Mexico City 14080, Mexico; 3Jurisdiccion 4, Secretaria de Salud de Michoacan, Patzcuaro 61600, Michoacan, Mexico; \\ ${ }^{4}$ Hospital Infantil de Morelia "Eva Samano de Lopez Mateos", Morelia 58000, Michoacan, Mexico
}

\begin{abstract}
Extra-enteric infections by Blastocystis spp. have rarely been documented. Here, we report a case of extra-enteric blastocystosis in a patient with minimal cervicitis symptoms. A 47-year-old Hispanic female patient was attended in a primary health centre in Michoacan state, Mexico, for her routine gynaecological medical examination. As only symptom, she referred to a slight vaginal itching. The presence of several vacuolar-stages of Blastocystis spp. were identified by Papanicolaou staining; molecular identification was attempted by culture-PCR sequencing of a region of $18 \mathrm{~S}$ gene from cervical and faecal samples obtained 2 months after cytological examination, even when patient declared that she tried selfmedicating with vaginal ovules. Blastocystis ST1 was identified only in the faecal sample. The presence of Blastocystis spp. in the cervix of a patient with scarce symptomatology, demonstrates the extraordinary flexibility of this microorganism to adapt to new environments and niches.
\end{abstract}

Key words: Blastocystis spp., Blastocystis subtypes, Papanicolaou stain, cervix, extra-enteric blastocystosis

\section{INTRODUCTION}

Blastocystis is a widespread intestinal eukaryote identified in human beings and several animals; its prevalence in humans ranges from $10 \%$ in developed countries to $>60 \%$ in developing ones. Four stages are presently recognized in Blastocystis: vacuolar, also named "central body"; granular; amoeboid; and cyst. Furthermore, 17 ribosomal lineages, known as subtypes (STs), have been described based on genotyping of the smallsubunit ribosomal RNA (18S rRNA) gene $[1,2]$. The clinical relevance of Blastocystis is still uncertain and even controversial. While some epidemiological and molecular data support a potential pathogenic role for this microorganism as an aetiological agent of dermatological and intestinal disorders [3-5], other studies indicate that Blastocystis is more commonly found in healthy individuals than in patients with gastrointestinal disorders $[6,7]$. The female genital tract can harbour a

- Received 9 Mar 2020, revised 24 August 2020, accepted 28 August 2020.

*Corresponding author (maravillap@yahoo.com)

(c) 2020, Korean Society for Parasitology and Tropical Medicine

This is an Open Access article distributed under the terms of the Creative Commons Attribution Non-Commercial License (https://creativecommons.org/licenses/by-nc/4.0) which permits unrestricted non-commercial use, distribution, and reproduction in any

medium, provided the original work is properly cited. variety of viral, bacterial, fungal and parasitic organisms. Bacterial cervicitis is the most common cause of these female genital tract infections, followed by viral and fungal cervicitis; however, only rare reports of cervix infections by intestinal protozoa have been described [8]. In present study, we report the presence of Blastocystis spp. colonizing in the cervix of a patient with scarce symptomatology.

\section{CASE RECORD}

This study was carried out in accordance with the recommendations of the guidelines of the Helsinki Declaration, and the Ethics and Research Committees of the General Hospital "Dr. Manuel Gea Gonzalez" approved the protocol (approval No. 12-77-2018). Written informed consent was obtained from the patient. A 47-year-old Hispanic female patient with a weight of $51 \mathrm{~kg}$ and height of $1.6 \mathrm{~m}$, she lives in a small rural village from the Michoacan state, Mexico (latitude: 19² 23'27"; longitude: $\left.101^{\circ} 63^{\prime} 39^{\prime \prime}\right)$, this community is placed inside an area with high poverty and difficult access [9]. Patient was attended in a primary health centre for her routine gynaecological medical examination. She was married with 4 children and her past medical history revealed unremarkable findings for 
chronic and hereditable diseases such as hypertension, type 2 diabetes mellitus, and Tb, HIV, and HPV infections (last, can be a relevant cause of cervicitis). The patient has elementary school and reported a complete vaccination scheme. During the medical interview, she denied addictions such as smoking, alcoholism and drugs. Regarding gynaecological information her menarche was at 16 years old with regular menstruation every 28 days, and she was currently without an active sex life. Initially, the patient did not report any symptoms; however, she later referred to a slight vaginal itching that began 2 months before the medical interview. A sample for cervical smear and cytological examination was obtained; during sampling, the posterior cervix exhibited slight inflammation with redness and abundant transparent secretion; no treatment was indicated to the patient. The cytological examination was performed by Papanicolaou stain (Pap) according to procedures stated in the Mexican guideline NOM-014-SSA2-1994, reporting non-specific inflammatory alteration with the absence of cells from the transformation zone. Interestingly, during the microscopic examination, several rounded cells, with a large central vacuole and a discrete peripheral cytoplasm with granule-like organelles located within thickened cytoplasmic regions, resembling Blastocystis spp. were observed (Fig. 1), even some of them were in cell division (Fig. 2).

The availability to contact the patient was low due to her residence place with communications shortage, almost 2 months after cytological examination, the patient was requested for new cervical and faecal samples. During the second clinical inter-
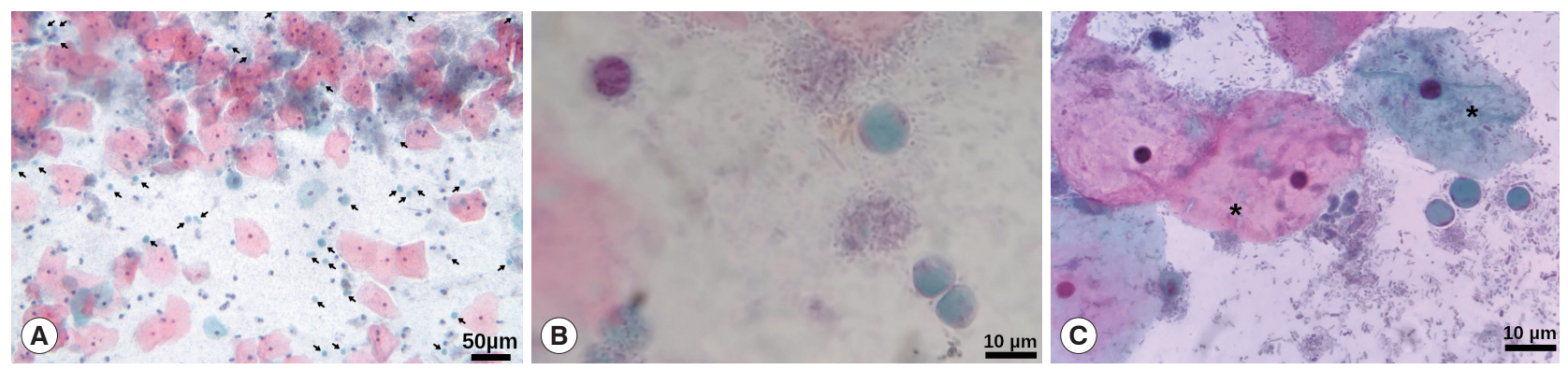

Fig. 1. Papanicolaou staining. (A) Magnification at $10 \times$, arrows point to many Blastocystis cells. Barr, $50 \mu$ m. (B, C) Magnification at $40 \times$, vacuolar stages of Blastocystis spp. Some typically superficial and intermediate squamous intraepithelial cells are also shown $\left(^{\star}\right)$. Barr, $10 \mu \mathrm{m}$.
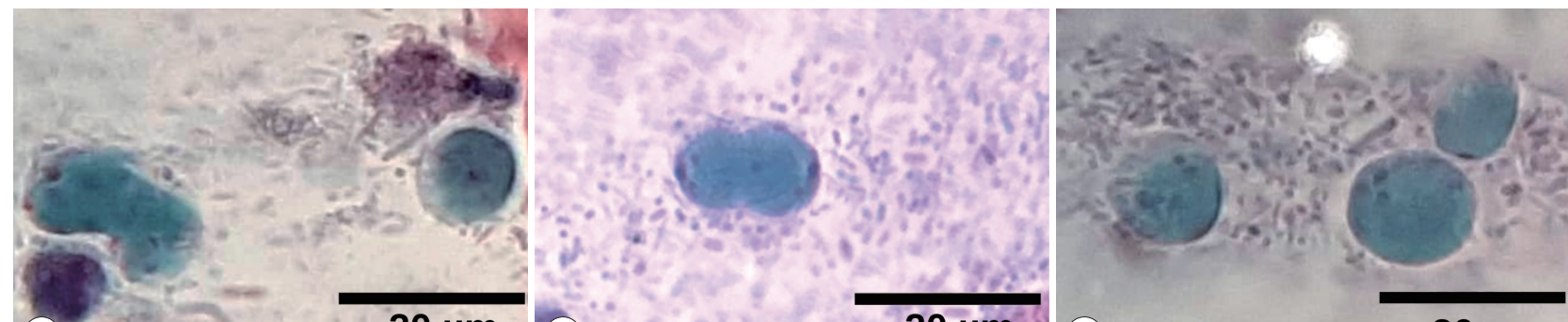

(A)
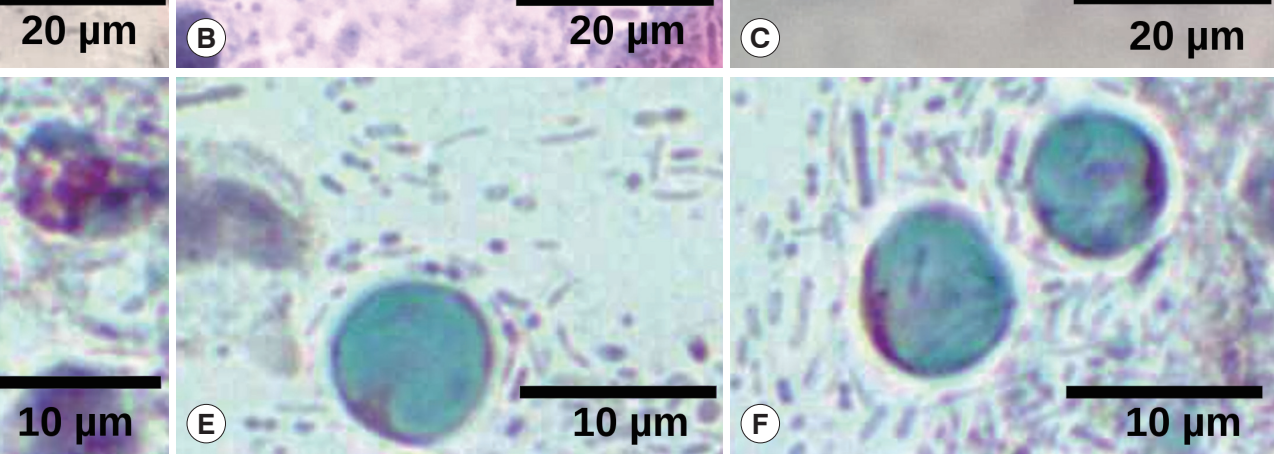

Fig. 2. Collage of different fields of Papanicolaou staining, highlighting several Blastocystis cells. (A, B) Cell division of Blastocystis vacuolar stages. (C-F) Some typically vacuolar stages of Blastocystis are shown. Magnification at 40x. Barr, $20 \mu \mathrm{m}$ for (A-C) and $10 \mu \mathrm{m}$ for (D-F). 
view, the patient did not refer any symptom but revealed that she tried self-medicating with vaginal tablets after first medical interview, though she could not remember the dose or name of the administered drug; cervical and faecal samples were placed in Blastocystis egg medium (ATCC 1671 medium) and sent to the laboratory; then, they were submitted to culture in vitro under anaerobic conditions using GasPak Jar at $37^{\circ} \mathrm{C}$. To rule out the presence of an intestinal fissure with drainage to the womb, an aliquot of the cervical sample was cultured in Chocolate agar, Columbia agar with 5\% Sheep Blood, MacConkey agar, Gardnerella agar and CHROMID ${ }^{\circledR}$ Candida agar plates; subsequently, some colonies were submitted to analysis by Vitek-MS MALDI-TOF technology (BioMérieux, Marcy l'Etoile, France).

After $72 \mathrm{hr}$ of culture, the sediments of cervical and faecal samples were processed to obtain DNA using a ZR Faecal DNA MiniPrep kit (Zymo Research, Irvine, California, USA) according to the manufacturer's protocol. Subtype identification was initially performed according to Santin et al. [10]. In addition, ST identification using the Pap smear was also attempted by PCRsequencing [11,12]. Amplicons were purified and sequenced in both directions by a commercial supplier. All sequences were subjected to a BLAST search of the GenBank database; multiple alignments were performed using the MUSCLE tool from the MEGA Program [13]. Phylogenetic reconstruction was inferred by Kimura's-2-parameter distance estimates [14], on the sequences obtained in the present study as well as those available in GenBank for 18S rRNA gene of Blastocystis ST1 to ST17. Bootstrap values were calculated by the analysis of 1,400 replicates from the neighbour-joining tree. During microscopic examination of Blastocystis, culture of both samples did not allow for the identification of parasitic structures. Only PCR for the faecal sample yielded an amplicon with a high identity to ST1 (99.6\%; compared with the reference strain Nand II of Blastocystis ST1, with accession number LXWW01000578), and the sequence was submitted to GenBank (accession number, MK932720); similarly, the phylogenetic tree located the sample in the clade for ST1 (Fig. 3). The microbiological cultures

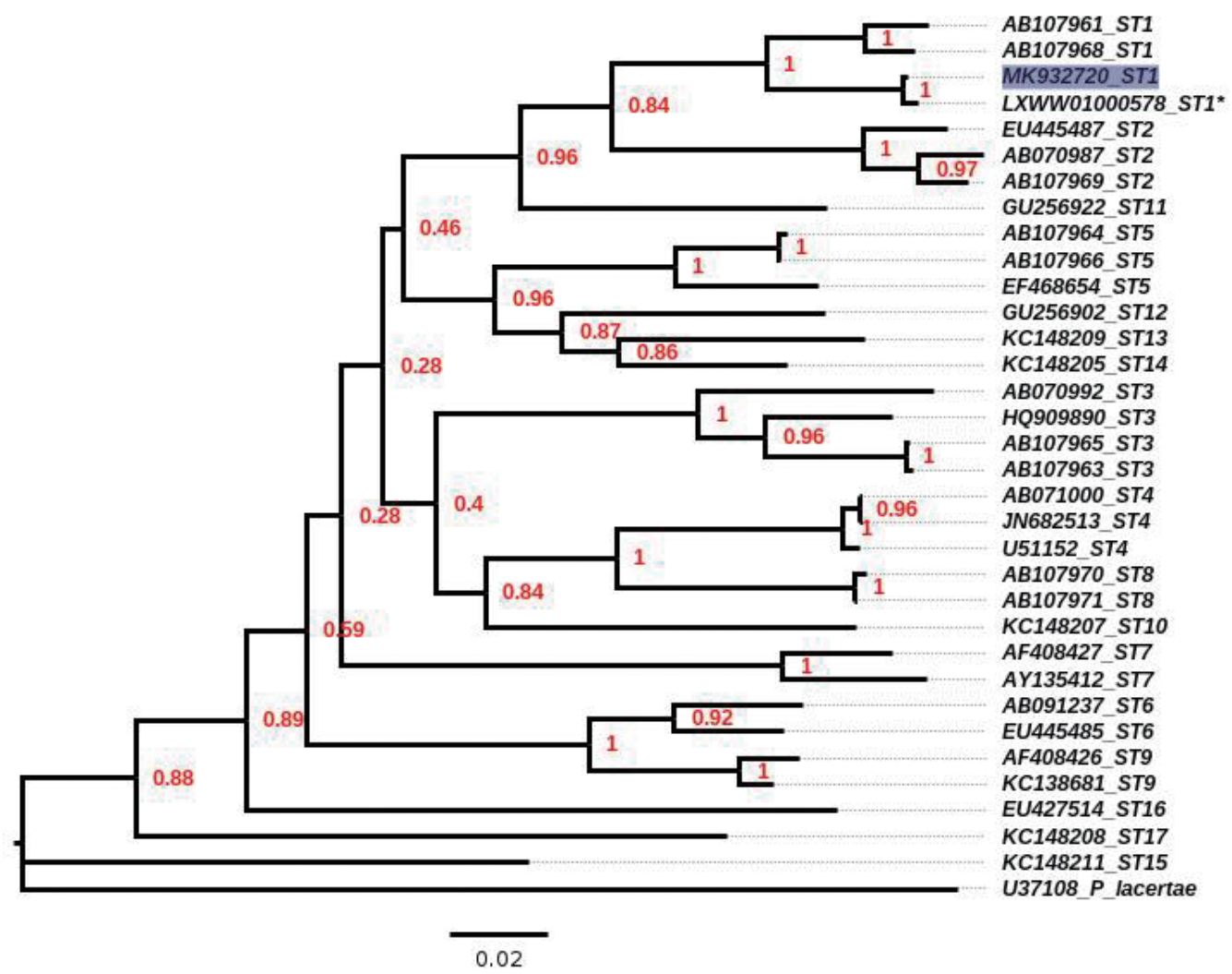

Fig. 3. Neighbour-joining tree, inferred by distance-based analysis of $18 \mathrm{~S}$ gene partial sequence of Blastocystis spp, using Kimura's-2parameter distance estimates. Sequences of Blastocystis subtypes (ST1-ST17) used for comparison were from GenBank database. *mean the sequence obtained from contig position from 92554 to 92993 by the reference strain ATCC 50177/Nand II. Scale bar shows 0.02 substitutions (corrected) per base pair. Shaded square indicates sequence obtained in the present study. Numbers in nodes mean the bootstrap values. 
for the cervical sample showed several colonies of Lactobacillus spp. and few colony-forming unit of Candida glabrata.

\section{DISCUSSION}

There are few cases of extra-enteric Blastocystosis in humans, one of which was a 29-year-old woman from Jamaica with rheumatoid arthritis taking prednisone. Several vacuolar forms of Blastocystis were found in synovial fluid from her left knee [15]. A 63-year-old man from Taiwan showed a large abscess in the right lobe of the liver that, during CT-guided percutaneous drainage, yielded chocolate-like pus, and the stained smear revealed the presence of vacuolar cells of Blastocystis [16]. Similarly, a Russian 64-year-old female patient developed a liver abscess with typical amoeboid and vacuolar stages of Blastocystis spp., some of them in the process of division [17]. In another report, the presence of Blastocystis vacuolar-like stage in a peritoneal fluid sample from a 46-year-old Hispanic woman with invasive, poorly differentiated adenocarcinoma and associated bowel perforation was documented [18]. Also, in a Brazilian 22-year-old female patient who presented splenic cysts, the cyst fluid analysis demonstrated numerous vacuolarstage

Blastocystis spp. cells, and PCR-sequencing analyses confirmed the presence of ST3 of this microorganism [19]. During a review, performed by Zierdt [20], he referred that Wolynska and Soroczan [21] examined 312 Polish peasant women for vaginal parasites. They reported Blastocystis with "various grades of invasion" in 47 women (11.5\%), in 16 of them this microorganism was found in the rectal area, in 22 cases in the vagina only, and in 9 cases in both rectal and vaginal areas. The women had vaginitis and cervical erosion. The authors attributed the disease to poor personal hygiene. Interestingly, in all previous reports and in the present study, the vacuolar stage has been clearly identified; this form is the most frequently observed in laboratory culture and in stool samples $[1,20,22]$, suggesting that this is the most feasible stage, for colonization of this microorganism. Due to patient used vaginal ovules, the culture and PCR for the cervical samples were negative, these products can be acquired without a medical prescription in Mexico, mainly those commercial ovules containing metronidazole.

On the other hand, during Pap smear, yeasts were not identified, because the sensitivity of this technique for diagnosis of Candida is low (25-52\%), but with high specificity (>89\%)
$[23,24]$ meaning that those cases in which yeasts are identified, are not misdiagnosed as another pathogen. In addition, the presence of C. glabrata as an opportunistic pathogen, without evident symptoms, suggests that the patient was immunocompetent [25]. In addition, due to the patient only exhibited minimal cervicitis symptoms and there are technical limitations in many of the local primary health centres in Mexico, mainly to develop molecular assays; the diagnosis of Chlamydia trachomatis, Mycoplasma genitalium, HSV type 2, EBV, or CMV was not made.

The patient was carried out of Blastocystis ST1, this is a common subtype in Mexico and South America [26-28]. It has been reported that ST1 can colonize the enteric tract of several animals, including birds, pigs, cows, dogs, rats and non-human primates $[29,30]$. A recent study showed that ST1 exhibits a generalist profile with minimal differentiation between parasite populations, as well as substantial gene flow between them, suggesting that there are a single ST1 metapopulation i.e., ST1 is an ensemble of highly interacting populations between and within them [30].

It is not clear how Blastocystis reached to the cervix because the patient referred did not to have an active sex life and did not exhibit immunocompromise or a chronic infection; there was also no evidence of an intestinal fissure that could drain stool into the womb. According to findings reported by Wolynska and Soroczan [21], an anal-genital transmission during incorrect cleaning of the genital area could have been carried out in our patient; besides, the stool culture and PCR data targeting Blastocystis in this patient can be helpful to suggest the anal-genital transmission. In conclusion, although the reports of extra-enteric Blastocystosis are scarce, this case as a few others, demonstrates the extraordinary flexibility of Blastocystis spp.to adapt to new environments and niches.

\section{ACKNOWLEDGMENTS}

Gloria Alicia Figueroa-Aguilar, Wendy Vianey Padilla-Cabrera and Nelly Raquel Gonzalez- Arenas for their technical assistance. To Laura Marquez and Nelly Lopez, LaNaBio, Instituto de Biologia, UNAM for their assistance during sequencing technique. This work was partially supported by the Consejo Nacional de Ciencia y Tecnologia (CONACYT) grant CB-2011/ 168619 . 


\section{CONFLICTS OF INTEREST}

The authors declare that they have no conflicts of interest.

\section{REFERENCES}

1. Tan KSW. New insights on classification, identification and clinical relevance of Blastocystis spp. Clin Microbiol Rev 2008; 21: 639665.

2. El Safadi D, Gaayeb L, Meloni D, Cian A, Poirier P, Wawrzyniak I, Delbac F, Dabboussi F, Delhaes L, Seck M, Hamze M, Riveau G, Viscogliosi E. Children of Senegal River Basin show the highest prevalence of Blastocystis sp. ever observed worldwide. BMC Infect Dis 2014; 14: 1-11.

3. Yakoob J, Jafri W, Jafri N, Khan R, Islam M, Beg MA, Zaman V. Iritable Bowel Syndrome: in search of an etiology: role of Blastocystis hominis. Am J Trop Med Hyg 2004; 74: 383-385.

4. Turkeltaub JA, McCarty TR 3rd, Hotez PJ. The intestinal protozoa: emerging impact on global health and development. Curr Opin Gastroenterol 2015; 31: 38-44.

5. Jimenez-Gonzalez DE, Martinez-Flores WA, Reyes-Gordillo J, Ramirez-Miranda ME, Arroyo-Escalante S, Romero-Valdovinos M, Stark D, Souza-Saldivar V, Martinez-Hernandez F, Flisser A, Olivo-Diaz A, Maravilla P. Blastocystis infection is associated with Irritable Bowel Syndrome in a mexican patient population. Parasitol Res 2012; 110: 1269-1275.

6. Scanlan PD. Blastocystis: past pitfalls and future perspectives. Trends Parasitol 2012; 28: 327-334.

7. Stensvold CR, Van der Giezen M. Associations between gut microbiota and common luminal intestinal parasites. Trends Parasitol 2018; 34: 369-377.

8. Calore EE, Calore NMP, Cavaliere MJ. Salpingitis due to Entamoeba histolytica. Braz J Infect Dis 2002; 6: 97-99.

9. Consejo Nacional de Evaluación de la Política de Desarrollo Social (CONEVAL). MEDICIÓN DE LA POBREZA [Internet]; [cited 2019 October 29]. Available from: https://www.coneval.org.mx/ Medicion/Paginas/Pobrezalnicio.aspx.

10. Santín M, Gómez-Muñoz MT, Solano-Aguilar G, Fayer R. Development of a new PCR protocol to detect and subtype Blastocystis spp. from humans and animals. Parasitol Res 2011; 109: 205-212.

11. Sepp R, Szabó I, Uda H, Sakamoto H. Rapid techniques for DNA extraction from routinely processed archival tissue for use in PCR. J Clin Pathol 1994; 47: 318-323.

12. Feine I, Shpitzen M, Geller B, Salmon E, Peleg T, Roth J, Gafny R. Acetone facilitated DNA sampling from electrical tapes improves DNA recovery and enables latent fingerprints development. Forensic Sci Int 2017; 276: 107-110.

13. Edgar RC. MUSCLE: multiple sequence alignment with high accuracy and high throughput. Nucleic Acids Res 2004; 32: 17921797.

14. Kumar S, Stecher G, Li M, Knyaz C, Tamura K. MEGA X: molecular evolutionary genetics analysis across computing platforms. Mol
Biol Evol 2018; 35: 1547-1549.

15. Lee MG, Rawlins SC, Didier M, DeCeulaer K. Infective arthritis due to Blastocystis hominis. Ann Rheum Dis 1990; 49: 192-193.

16. Hu KC, Lin CC, Wang TE, Liu CY, Chen MJ, Chang WH. Amoebic liver abscess or is it? Gut 2008; 57: 627.

17. Prodeus TV, Zeila OP, Khlebnikova TA, Pikul' DA. Extraenteric infection caused by Blastocystis spp. in a female patient with liver abscess. Med Parazitol (Mosk) 2014; 2: 6-9 (in Russian).

18. Patino WD, Cavuoti D, Banerjee SK, Swartz K, Ashfaq R, Gokaslan T. Cytologic diagnosis of Blastocystis hominis in peritoneal fluid: a case report. Acta Cytol 2008; 52: 718-720.

19. Santos HL, Sodré FC, de Macedo HW. Blastocystis sp. in splenic cysts: causative agent or accidental association? A unique case report. Parasit Vectors 2014; 7: 207.

20. Zierdt CH. Blastocystis hominis--past and future. Clin Microbiol Rev 1991; 4: 61-79.

21. Wolyńska M, Soroczan W. Blastocystis hominis Brumpt, 1912, (Phycomycetes) in the female genital tract. Pol Tyg Lek 1972; 27: 788-791.

22. Stensvold CR, Nielsen HV, Mølbak K, Smith HV. Pursuing the clinical significance of Blastocystis diagnostic limitations. Trends Parasitol 2009; 5: 23-29.

23. Engberts MK, Goedbloed AF, van Haaften M, Boon ME, Heintz PM. Microscopic diagnosis of vulvovaginal candidiasis in stained vaginal smears by Dutch general practitioners. Acta Cytol 2007; 51: 882-885.

24. Avwioro Og, Olabiyi Oe, Avwioro To. Sensitivity of a papanicolaou smear in the diagnosis of Candida albicans infection of the cervix. N Am J Med Sci 2010; 2: 97-99.

25. Kumar K, Askari F, Sahu MS, Kaur R. Candida glabrata: a lot more than meets the eye. Microorganisms 2019; 7: 39.

26. Villalobos G, Orozco-Mosqueda GE, Lopez-Perez M, LopezEscamilla E, Córdoba-Aguilar A, Rangel-Gamboa L, Olivo-Diaz A, Romero-Valdovinos M, Maravilla P, Martinez-Hernandez F. Suitability of internal transcribed spacers (ITS) as markers for the population genetic structure of Blastocystis spp. Parasit Vectors 2014; 7: 461.

27. Villegas-Gómez I, Martínez-Hernández F, Urrea-Quezada A, González-Díaz M, Durazo M, Hernández J, Orozco-Mosqueda GE, Villalobos G, Maravilla P, Valenzuela O. Comparison of the genetic variability of Blastocystis subtypes between human carriers from two contrasting climatic regions of México. Infect Genet Evol 2016; 44: 334-340.

28. Ramírez JD, Sánchez A, Hernández C, Flórez C, Bernal MC, Giraldo JC, Reyes P, López MC, García L, Cooper PJ, Vicuña Y, Mongi F, Casero RD. Geographic distribution of human Blastocystis subtypes in South America. Infect Genet Evol 2016; 41: 3235.

29. Yoshikawa H, Wu Z, Nagano I, Takahashi Y. Molecular comparative studies among Blastocystis isolates obtained from humans and animals. J Parasitol 2003; 89: 585-594.

30. Villanueva-Garcia C, Gordillo-Chavez EJ, Lopez-Escamilla E, Rendon-Franco E, Muñoz-Garcia CI, Gama L, Martinez-Flores 
WA, Gonzalez-Rodriguez N, Romero-Valdovinos M, Diaz-Lopez H, Galian J, Villalobos G, Maravilla P, Martinez-Hernandez F. Clarifying the cryptic host specificity of Blastocystis spp. isolates from Alouatta palliata and A. pigra howler monkeys. PLoS One 2017; 5: 12: e0169637. 